tren läßt den Schluß zu, daß die Strukturunterschiede der beiden cyclischen Trimeren in der Nachbarschaft des Ringstickstoffes zu suchen sind. Die = NH-Gruppe sowie ein Amin haben in beiden Fällen die gleiche Lage, während das Ring-NH sowie das zweite Amin unterschiedlich sind (II 5,2 $\tau$ bzw. $1,2 \tau ;$ IV $4,5 \tau$ bzw. $1,6 \tau)$.

Das UV-Spektrum der beiden ringgeschlossenen Trimeren zeigt eine sehr starke Abhängigkeit der Absorption vom $p_{\mathrm{H}}$-Wert des Lösungsmittels. Im alkalischen Medium sind die Absorptionen von II und IV sehr ähnlich (330 und $280 \mathrm{~nm}$ sowie 338 und $260 \mathrm{~nm}$ ), im neutralen Bereich liegen sie bei $340,280 \mathrm{~nm}$ und bei $350,320,240 \mathrm{~nm}$, während sie bei $p_{\mathrm{H}} 3$ bei $340,280 \mathrm{~nm}$ bzw. 350,320 und $240 \mathrm{~nm}$ gefunden werden. Auf Grund der schweren Löslichkeit sind die $\lg \varepsilon$-Werte nicht in allen Fällen anzugeben. Die Lage der Maxima bei 350, 320, $240 \mathrm{~nm}$ kann bei einem Vergleich mit dem Ring. system des 2-Azaindolizins als Stütze für den Strukturvorschlag angesehen werden ${ }^{8}$. Das Massenspek-

8 J. D. BowER, J. chem. Soc. [London] 1957, 4510. trum von IV weist folgende charakteristische Brückstücke auf, die als Hinweis für die angegebene Struktur gewertet werden:

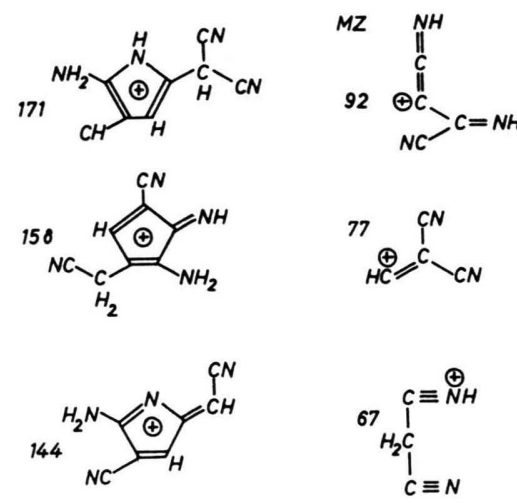

Eine Unterscheidung zwischen den Strukturisomeren von IV in bezug auf die gegenseitige Lage von Imin und Nitril in Stellung 1 und 2 ist kaum möglich, das Fragment der MZ 171 spricht jedoch dafür, daß die Nitrilgruppe sich in Position 1 befindet.

\title{
Chemilumineszenz von Mitochondrien
}

\author{
Joachim Stauff und Jörg Ostrowski \\ Institut für Physikalische Biochemie und Kolloidchemie im Institut für Physikalische Chemie \\ der Universität Frankfurt a. M. \\ (Z. Naturforschg. 22 b, 734-740 [1967]; eingegangen am 3. Dezember 1966)
}

\begin{abstract}
Mitochondrien aus Rattenleber (RL) und Rinderherzmuskel (BH) erzeugen bei Behandlung mit $\mathrm{O}_{2}$ eine schwache Chemilumineszenz in dem Spektralbereich zwischen 400 und $650 \mathrm{~m} \mu$, deren Intensität bei RL-Mitochondrien durch vorheriges Einfrieren und Auftauen wie durch Ultraschallbehandlung größer wird. Bei beiden Arten verursacht Zusatz von Acridinorange eine wesentliche Verstärkung der Lumineszenz, gleichzeitig wird die $\mathrm{O}_{2}$-Aufnahme gehemmt. RL-Mitochondrien erzeugen unter diesen Bedingungen eine kurzzeitige, BH-Mitochondrien eine langsam ansteigende langandauernde Strahlung; das gleiche Verhalten zeigen aus BH-Mitochondrien gewonnene „electrontransfer-particles" (ETP). Der zeitliche Ablauf und die Beeinflußbarkeit durch Effektoren der Atmungskette ist andersartig als bei der von Vhadimirov gefundenen Chemilumineszenz von RL-Mitochondrien. Als Träger der Lumineszenz wird angeregter $\mathrm{O}_{2}$ diskutiert.
\end{abstract}

In einer früheren Arbeit wurde von uns ${ }^{1}$ und inzwischen auch von anderer Seite ${ }^{2}$ beobachtet, daß in Rattenleberhomogenaten eine sehr schwache Lumineszenz auftritt, wenn diese mit $\mathrm{O}_{2}$ behandelt werden. Ähnliche Ergebnisse bei aerob wachsender Hefe $^{3,4}$ ließen die Vermutung aufkommen, daß die Ursache der Strahlung im Stoffwechsel der Mito-

1 J. Stauff u. H. Schmidkunz, Z. physik. Chem. N.F. 33, 273 [1962].

2 Yu. A. Vladimirov u. S. F. Lvova, „Biophysik der Zelle“, Akademieverlag, Moskau 1965.

3 J. Stauff u. G. Reske, Nature [London] 198, 281 [1963]. chondrien zu suchen sein könnte, da die Wellenlänge der beobachteten Lumineszenz kleiner als $650 \mathrm{~m} \mu$ (entsprechend $45 \mathrm{Kcal} / \mathrm{Mol}$ ) beträgt und daher nur von einer Reaktion mit großem Energieumsatz verursacht sein kann. Orientierende Versuche mit isolierten Mitochondrien aus Rattenlebern und Rinderherzen*, in wäßriger 0,25-m. Rohrzucker-

4 A. G. Gurwitsch u. L. D. Gurwitscir, „Die mitogenetische Strahlung“, Jena 1959.

* Im folgenden werden die Abkürzungen „RL“ für Rattenleber und „BH“ für Rinderherz-Mitochondrien gebraucht. 
lösung aufgeschwemmt, ließen nur eine äußerst geringe Lichtemission erkennen. Partielle Zerstörung der Einheiten mit Hilfe von Ultraschall oder durch Einfrieren und Auftauen ließ einen Mechanismus wirksam werden, der bei Zufuhr von $\mathrm{O}_{2}$ eine schwache, aber gut zu beobachtende Lumineszenz hervorrief. Eine nicht unerheblich stärkere Lichtemission wurde hingegen beobachtet, wenn Mitochondrien oder Electron-Tranfer-Partikeln (nach Green $^{5}$ ) geringe Mengen des Farbstoffs Acridinorange zugesetzt werden ${ }^{6}$. Auch bei den partiell zerstörten Mitochondrien bewirkt Acridinorange eine Verstärkung, doch dauert diese nur einige Min., während das Leuchten der intakten BH-Mitochondrien mit Acridinorange mehrere Stdn. anhält.

Von Vladimirov und Mitarbb. ${ }^{7}$ wurde inzwischen eine ähnliche Lichtemission an RL-Mitochondrien beobachtet, die ebenfalls von langer Dauer ist $(0,5$ bis 2 Stdn.). Diese Lumineszenz soll im Zusammenhang mit einer Lipoid-Peroxidation stehen und mit der oxidativen Phosphorylierung gekoppelt sein. Bei unseren Beobachtungen traten zwei durch ihren Zeitablauf deutlich unterscheidbare Leuchtreaktionen auf, die sich durch Inhibitoren wie KCN, Antimycin, 2.4-Dinitrophenol u. a. nicht hemmen ließen, doch bei der Denaturierung durch Desoxycholat $(0,3 \%)$ unterdrückt wurden. Die von Vladimirov und Mitarbb. ${ }^{7}$ beobachtete Lichtemission wurde hingegen durch die genannten Inhibitoren mehr oder weniger unterdrückt; Erhitzen der Mitochondrien-Suspension setzte das Strahlungsmaximum zwar auf die Hälfte herab, veränderte die im gesamten Zeitablauf emittierte Strahlung hingegen kaum. Danach hat es den Anschein, als ob die von Vladimirov und Mitarbb. gefundenen Effekte mit den von uns beobachteten nicht in direktem Zusammenhang stehen und mit mehr als einer Ursache gerechnet werden muß, die bei Mitochondrien zur Bildung angeregter Zustände führt. Für die unterschiedlichen Befunde mögen auch die verschiedenen Versuchsbedingungen verantwortlich sein.

Da die bei den Mitochondrien und ETP** beobachteten Lichtemissionen in jedem Fall nur bei Zufuhr von $\mathrm{O}_{2}$ auftreten, sollten diese mit denjenigen Lichterscheinungen im Zusammenhang gebracht

5 D. E. Green, Biochim. biophysica Acta [Amsterdam] 23, 516 [1957].

6 J. Stauff, Ber. Bunsenges. physik. Chem. 68, 773 [1963].

7 Yu. A. Vladimirov, Biokhimiya 31, 507 [1966]. werden können, die bei vielen anorganischen und organischen Oxidationsprozessen auftreten und über die bereits früher berichtet worden ist ${ }^{1,8}$. Bei diesen Prozessen treten zumeist intermediär Sauerstoffradikale auf $\left(\mathrm{OH}, \mathrm{O}_{2} \mathrm{H}, \mathrm{O}_{2}{ }^{\ominus}\right)$, deren Reaktionen zu angeregtem $\mathrm{O}_{2}$ im ${ }^{1} \Delta$ - oder ${ }^{1} \sum$-Zustand - sogenanntem Singulett- $\mathrm{O}_{2}$ führen, der schließlich für die Leuchterscheinungen verantwortlich ist. Diese Frage ist deswegen von Interesse, weil vielfach bestritten wird, daß in biologischen Systemen Akkumulationen der Energie auf Beträge von über $50 \mathrm{Kcal} / \mathrm{Mol}$ auftreten können, obwohl das Vorhandensein einer Biolumineszenz als solche allein schon dagegen spricht. Eine solche Speicherung in einem Molekül ist bei biologischen Reaktionen sicherlich nicht in einem einzigen Reaktionsschritt möglich. Bei zwei oder mehr Schritten ist sie möglich, wenn die energiereichen Zwischenprodukte eine hinreichend lange Lebensdauer haben. So konnte durch die reaktionskinetische Analyse ${ }^{9}$ der bei der Reaktion zwischen $\mathrm{Ce}^{4 \oplus}$ und $\mathrm{H}_{2} \mathrm{O}_{2}$ auftretenden Chemilumineszenz nachgewiesen werden, daß die Strahlungsenergie von mehr als $45 \mathrm{Kcal} / \mathrm{Mol}$ durch Stoßkomplexe zweier Singulett-Sauerstoffmoleküle mit jeweils 20-35 Kcal/Mol Energie oberhalb des Grundzustandes geliefert wird. Jedes dieser Moleküle braucht aber mindestens 2 Moleküle $\mathrm{H}_{2} \mathrm{O}_{2}$ zu seiner Bildung. Ein Quant der endgültig emittierten Strahlung bezieht seine Energie also von der Oxydation von 4 Molekülen $\mathrm{H}_{2} \mathrm{O}_{2}$ durch $\mathrm{Ce}^{4 \oplus}$-Ionen.

Wegen dieser Ergebnisse scheint es nicht von vornherein unmöglich zu sein, entsprechende Reaktionen auch bei biologischen Prozessen zu suchen, um so mehr, als eine Reihe von Experimenten zeigt, daß auch einfache anorganische wie organische Substanzen, die katalytisch durch Luftsauerstoff oxidiert werden können, Lumineszenzerscheinungen hervorriefen ${ }^{9}$. Die untere Grenze der beobachtbaren Strahlungen liegt bei $650 \mathrm{~m} \mu$, die durch die Empfindlichkeitsgrenze des verwendeten Photomultipliers gegeben ist. Die strahlenden Spezies müssen daher mindestens $45 \mathrm{Kcal} / \mathrm{Mol}$ gespeichert haben.

Im folgenden sollen die Einzelheiten der Beobachtungen an RL- und BH-Mitochondrien-Suspensionen mitgeteilt werden.

\footnotetext{
** ETP = Abkürzung für Elektronen-Transfer-Partikel nach GREEN ${ }^{5}$.

8 J. Stauff, Photochem. Photobiol. 4, 1199 [1965].

9 J. Stauff, Z. physik. Chem. N.F. 49, 58 [1966].
} 


\section{Experimenteller Teil}

1. Darstellung von Mitochondrien und Elektronen-Transport-Partikeln

a) RL-Mitochondrien wurden nach der Methode von SCHNEIDER ${ }^{10}$ präpariert. Aufbereitungszeit: 2 Stunden.

b) BH-Mitochondrien wurden nach einer modifizierten Vorschrift von CRANE und Mitarb. ${ }^{11}$ isoliert.

$450 \mathrm{~g}$ frische Rinderherzmuskeln wurden zerkleinert, zweimal durch einen vorgekühlten Fleischwolf geschickt und der Fleischbrei in $1-1,2 l$ einer 0,2-m. Rohrzuckerlösung, $0,185 \% \mathrm{KH}_{2} \mathrm{PO}_{4}$ enthaltend, aufgeschwemmt. Die Homogenisierung der Suspension wurde in einem vorgekühlten $1,5 l$-Starmix (Firma Bosch, unbelastet $13000 \mathrm{U} / \mathrm{min}$ ) vorgenommen, Zeitdauer: 60 Sekunden. Der $p_{\mathrm{H}}$-Wert wurde auf $7,2-7,4$ eingestellt und nach jeder Operation kontrolliert. Das Homogenat wurde sofort in einer Zentrifuge (Fa. Stock, Marburg) bei 2600 bis $2900 \mathrm{U} / \mathrm{min} 13$ Minuten zentrifugiert und der Überstand durch ein Leinentuch zur Entfernung von Fettbestandteilen filtriert. Das Sediment der Kernfraktion wurde verworfen und der Überstand portionsweise in einer MSE-Zentrifuge (Fa. Measuring \& Scientific Equipment, London) bei $17000 \mathrm{U} / \mathrm{min}(34000 \mathrm{~g}) 15$ Minuten zentrifugiert. Das Gesamtsediment wurde in $180 \mathrm{ml}$ Rohrzuckerlösung $(0,25 \mathrm{~m})$ suspendiert und erneut zentrifugiert. Der Überstand wurde verworfen. Dauer des Verfahrens: 6 Stunden.

c) Elektronen-Transport-Partikel (ETP) wurden nach der Alkohol-Phosphat-Methode von GreEN und Mitarb. $^{5}$ aus der L-Fraktion von BH-Mitochondrien nach Hatefi und Mitarb. ${ }^{12}$ dargestellt.

\section{A cetonbehandlung der Mito- chondrien}

BH-Mitochondrien wurden nach der Methode von Lester und Mitarb. ${ }^{13}$ mit Aceton extrahiert. Ubichinon und $2 / 3$ der Phospholipoide sollen dadurch entfernt werden, doch die Mitochondrien in ihrer morphologischen Struktur, wenn auch geschrumpft, erhalten bleiben.

\section{Ultraschallbehandlung}

Mitochondrien-Suspensionen wurden (in einem verschließbaren Wägegläschen) in einem Ölbad unter Anwendung einer elektrischen Leistung von 30 Watt 2 bis 6 Min. mit Ultraschall behandelt. Der Vorgang wurde häufig unterbrochen, um die Suspensionen in einem bereitstehenden Eisbad zu kühlen.

Die Präparate $(\mathrm{a}-\mathrm{c})$ wurden bei $+2{ }^{\circ} \mathrm{C}$ unter nicht sterilen Bedingungen im Kühlraum hergestellt, auf $18{ }^{\circ} \mathrm{C}$ in $0,25-m$. Rohrzuckerlösung eingefroren

10 W. C. Schneider, J. biol. Chemistry 176, 259 [1948].

11 F. L. Crane, Biochim. biophysica Acta [Amsterdam] 23, 516 [1957].

12 Y. Hatefi, Biochim. biophysica Acta [Amsterdam] 27, 84 [1958]. und im Dunkeln aufbewahrt. Gehaltsbestimmungen wurden auf der Basis eines Totalproteingehalts nach Gornall und Mitarb. ${ }^{14}$ vorgenommen. Der Totalproteingehalt wurde als „Biuret-Wert“ angegeben. Insgesamt wurden Mitochondrien dreimal aus Rattenleber und sechsmal aus Rinderherzmuskel aufgearbeitet und untersucht.

\section{Meßtechnik}

Die Lichtemission der Präparate wurde nach dem Prinzip von Stauff und Mitarbb. ${ }^{1,3,8}$ in einer Chemilumineszenz-Apparatur mit temperierbarer Küvette bei konstanter Temperatur $\left(35^{\circ} \mathrm{C}\right)$ gemessen. Als Empfänger diente ein Photomultiplier 6255 B (EMI, Electronics Ltd.) mit einem Empfindlichkeitsmaximum zwischen 400 und $600 \mathrm{~m} \mu$ (Betriebsspannung: $1380 \mathrm{~V}$, Dunkelstrom: $\left.1 \cdot 10^{-9} \mathrm{~A}\right)$. Das emittierte Licht wurde nicht spektral zerlegt; die Messungen wurden in einem verdunkelten Raum vorgenommen. Vor den Messungen wurde das Reaktionsgefäß mit Chromschwefelsäure und bidest. Wasser gereinigt und in den Thermostaten eingebaut. Dann wurden $5 \mathrm{ml}$ Phosphatpuffer $\left(p_{\mathrm{H}}\right.$ $7,7 \pm 0,1, \mathrm{~m} / 15)$ vorgelegt und auf $35^{\circ} \mathrm{C}$ erwärmt. Stickstoff bzw. Sauerstoff wurden nach Bedarf in das Reaktionsgefäß eingeleitet. Die auftretende Lichtemission wurde von einem Kompensationsschreiber (Philips, Hamburg) nach Verstärkung durch einen ZerhackerVerstärker (Knick, Berlin) aufgezeichnet. Für alle wäßrigen Lösungen und Suspensionen wurde bidest. Wasser aus einer Quarzapparatur verwandt. Reinststickstoff wurde nach Vorreinigung mit Pyrogallol bei $110{ }^{\circ} \mathrm{C}$ am Kupferkontakt (BTS-Katalysator, BASF) vom $\mathrm{O}_{2}$ bis auf $0,1 \mathrm{ppm}$ befreit. Acridinorange (Merck, Darmstadt) wurde als $\mathrm{ZnCl}_{2}$-Doppelsalz verwandt und enthielt Spuren von Eisen. (Diese Schwermetallionen hatten, wie gesonderte Versuche erwiesen, keinen Einfluß auf die Chemilumineszenz.) Die ChemilumineszenzEffekte eines Mitochondrien-Präparates waren innerhalb der Meßgenauigkeit reproduzierbar, verschiedene Präparate gleicher biologischer Herkunft zeigten den gleichen Gang im Strahlungsverhalten. Die Atmung der Mitochondrien in Gegenwart von Na-Succinat wurde durch Bestimmung der Geschwindigkeit der Abnahme der $\mathrm{O}_{2}$-Konzentration vorgenommen. Letztere wurde mit einem $\mathrm{O}_{2}$-Analysator Modell 777 (Firma Beckman Instr.) am offenen System bestimmt.

\section{Ergebnisse}

\section{Chemilumineszenz von Rattenlebermitochondrien}

Qualitative Vorversuche mit einer Fraktion von RL-Mitochondrien brachten die ersten Hinweise auf eine schwache Lichtemission in subzellularen Ein-

13 R. L. Lester, Biochim. biophysica Acta [Amsterdam] 47, 358 [1961].

14 A. G. Gornall, J. biol. Chemistry 177, 751 [1949]. 
heiten. Die Chemilumineszenz war abhängig von der $\mathrm{O}_{2}$-Zufuhr, dem Alter der Suspension und nahm mit steigender Temperatur zu (Abb. 1). Sie konnte mit Nicotinamid-adenin-dinucleotid $\left(\mathrm{NAD}^{\oplus}\right)$ verstärkt werden. Reduziertes Nicotinamid-adenin-dinucleotid

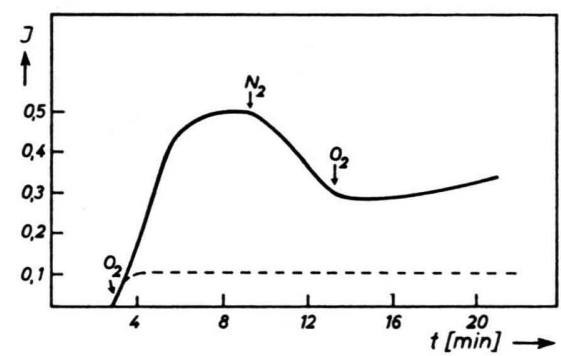

Abb. 1. Einfluß von $\mathrm{O}_{2}, \mathrm{~N}_{2}$ und Alter auf die „natürliche“ Chemilumineszenz von RL-Mitochondrien in 0,25-m. Rohrzuckerlösung. I = Intensität [Nanoampère]. - - 1 Tag alte Suspension, $\longrightarrow 30$ Tage alte Suspension.

(NADH) und Natriumsuccinat hatten keinen Einfluß. Wesentlich deutlicher wurde die Lichtemission, wenn die Mitochondrien bzw. deren Membranen durch Kältebehandlung und Ultraschall partiell zerstört wurden (Tab. 1).

\begin{tabular}{cc}
\hline $\begin{array}{c}I s \\
\text { Nanoampère/mg } \\
\text { Totalprotein] }\end{array}$ & $\begin{array}{c}\text { Aufbewahrungszeit } \\
\text { bei }-18^{\circ} \mathrm{C} \\
{[\text { Tage }]}\end{array}$ \\
\hline $0,06 \pm 0,01$ & - \\
$0,12 \pm 0,01$ & 2 \\
$0,24 \pm 0,02$ & 4 \\
$0,40 \pm 0,04^{*}$ & $4^{*}$ \\
$0,47 \pm 0,05$ & 8 \\
$0,57 \pm 0,06$ & 12
\end{tabular}

Tab. 1. Abhängigkeit der spezifischen Strahlungsintensität $I s$ von RL-Mitochondrien von der Vorbehandlung. * 4 Tage alte Mitochondrien-Suspension; 2 Min. mit Ultraschall behandelt.

Einfrieren auf $-18{ }^{\circ} \mathrm{C}$ und Wiederauftauen wirkt nach Lusena ${ }^{15}$ wie eine starke Erhöhung der Rohrzuckerkonzentration, wobei Membranteile zerstört werden, im Mitochondrion enthaltene Enzyme in den äußeren Rahmen austreten und bestimmte vorher unzugängliche Enzyme substratwirksam werden. Andererseits soll nach Greiff, Myers und PrIVITERA ${ }^{16}$ bei dieser Behandlung die Fähigkeit zur oxydativen Phosphorylierung abnehmen.

Die Intensität dieser „natürlichen“ Lichtemission der Mitochondrien reagiert auf das Einfrieren mit

15 C. V. Lusena, Can. J. Biochem. 43, 1787 [1965].

16 D. Greiff, M. Myers u. C. A. Privitera, Biochim. biophysica Acta [Amsterdam] 50, 233 [1961]. einer Zunahme, und zwar wird diese im Zeitraum von 12 Tagen laufend größer, wie aus Tab. 1 zu erkennen ist. Bei diesem Experiment bezieht sich die Zeitangabe auf die Zeit, die das Präparat im eingefrorenen Zustand verbracht hat. Die $\mathrm{O}_{2}$-Aufnahme in Gegenwart von Na-Succinat nahm bei den gleichen Proben im Lauf der Zeit annähernd linear ab.

Die Vermutung, daß die Intensität der Strahlung mit Veränderungen der Mitochondrien zusammenhängt, konnte durch zwei weitere Versuche gestützt werden. Wird eine Charge RL-Mitochondrien in zwei Teilfraktionen (A) und (B) halbiert, (A) konstant auf $-18{ }^{\circ} \mathrm{C}$ eingefroren, (B) dagegen täglich bei $0-4{ }^{\circ} \mathrm{C}$ aufgetaut und wieder auf $-18{ }^{\circ} \mathrm{C}$ abgekühlt, so zeigt (B) nach 9 Tagen eine um den Faktor 10 stärkere Chemilumineszenz als (A). Im gleichen Zeitraum war die $\mathrm{O}_{2}$-Aufnahme von (B) auf den Wert Null abgesunken. Wegen der in früheren Versuchen beobachteten verstärkenden Wirkung fluoreszierender Farbstoffe wie Acridinorange (AO) und Eosin auf die Chemilumineszenz von Oxydationsreaktionen ${ }^{17}$ wurden davon geringe Mengen dem Reaktionsmedium zugesetzt. Vom AO ist bekannt, daß es in den Stoffwechsel der Mitochondrien eingreift. Eigene Beobachtungen konnten bestätigen, da $\beta$ die $\mathrm{O}_{2}$-Aufnahme durch AO $\left(5 \cdot 10^{-5}-m\right.$.) in Gegenwart von Na-Succinat völlig unterbunden wird. Abb. 2 zeigt den verstärkenden Einfluß von AO auf die Lumineszenz der RL-Mitochondrien. Eosin bewirkt im Gegensatz zu AO in Konzentrationen von $5 \cdot 10^{-4}-m$. und $5 \cdot 10^{-6}-m$. Schwächung der Emission auf $2 / 3$ des Wertes der Ausgangsintensität.

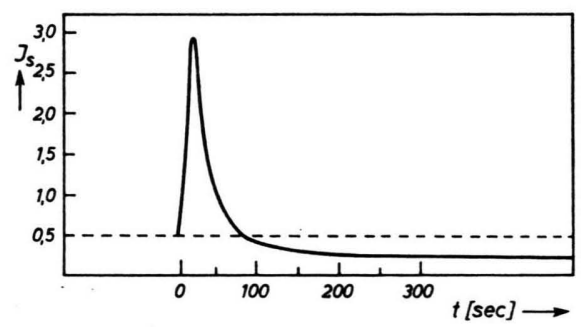

Abb. 2. Verstärkung der Chemilumineszenz RL-Mitochondrien durch Acridinorange $\left(1,8 \cdot 10^{-4}-m\right.$.). Is $=$ spezifische Intensität [Nanoampère/mg Totalprotein]. - - - ohne Acridinorange, — nach Zusatz von Acridinorange zum Zeitpunkt Null.

17 A. J. Zhuravlev, Biofizika 9, 671 [1964]. 


\section{Chemilumineszenz von Mitochondrien aus Rinderherzmuskel}

Obwohl BH-Mitochondrien unter den gleichen Bedingungen wie RL-Mitochondrien untersucht wurden, zeigten sich charakteristische Unterschiede in den Ergebnissen. Es wäre zu erwarten gewesen, daß $\mathrm{BH}$-Mitochondrien wegen ihrer gröberen Behandlung bei der Aufarbeitung nach Crane und Mitarb. eine stärkere „natürliche“ Chemilumineszenz zeigten als RL-Mitochondrien. Das Gegenteil war der Fall. Die „natürliche“ Strahlung war im Bereich der Meßgenauigkeit praktisch gleich Null und fast unabhängig vom Alterszustand bei Aufbewahrung bei $-18{ }^{\circ} \mathrm{C}$. Die $\mathrm{O}_{2}$-Aufnahme in Gegenwart von Succinat war am Tage der Aufarbeitung der BH-Mitochondrien um eine Zehnerpotenz höher als die der RL-Mitochondrien. Die aus Rinderherzmuskel isolierten ETP zeigten ebenfalls außerhalb der Fehlergrenze keine „natürliche“ Strahlung, obwohl ihre $\mathrm{O}_{2}$-Aufnahme um 50 bis $60 \%$ gegenüber der der BH-Mitochondrien erhöht war.

Ultraschallbehandlung hatte ebenfalls keinen Einfluß auf die Strahlungsintensität. Lumineszenz zeigten die BH-Mitochondrien und ETP erst bei Zusatz

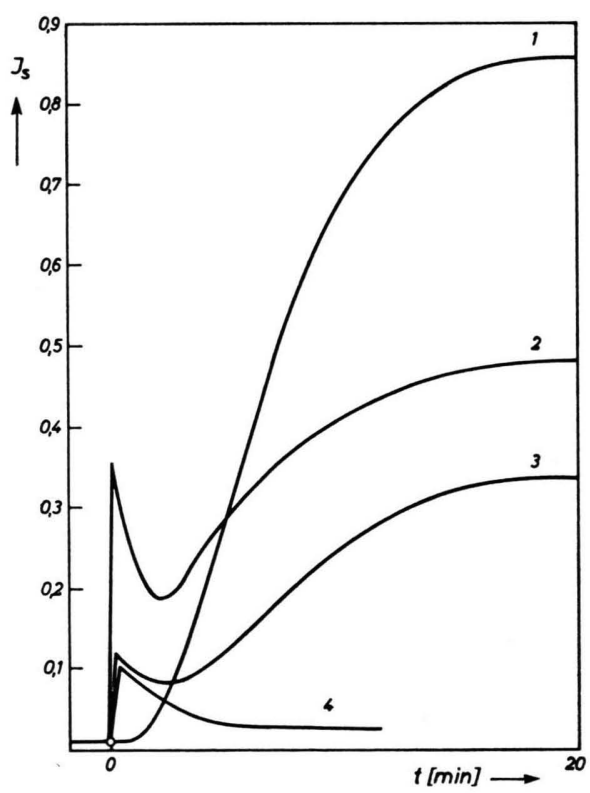

Abb. 3. Chemilumineszenz von BH-Mitochondrien und ETP in Gegenwart von Acridinorange $\left(1,8 \cdot 10^{-4}-m\right.$.). Is = spezifische Intensität [Nanoampère/mg Totalprotein]. Zur Zeit „Null“ Zugabe des Farbstoffs. (1) BH-Mitochondrien am Tage der Aufarbeitung, (2) BH-Mitochondrien, 7 Tage später, (3) ETP aus BH-Mitochondrien in Gegenwart von Na-desoxycholat $(0,3 \%)$ am Tage der Aufarbeitung. von Acridinorange. Die frisch dargestellten Mitochondrien erzeugten eine innerhalb von 20 Min. allmählich ansteigende Lumineszenz (vgl. Kurve 1, Abb. 3), die dann über mehrere Stdn. anhielt. Nach der Einfrierbehandlung traten bei einem 7 Tage alten Präparat wie bei den RL-Mitochondrien zunächst das kurzzeitige scharfe Maximum auf (Kurve 2, Abb. 3), danach jedoch entwickelte sich die langdauernde Strahlung, wenn auch nur noch mit etwa der halben Intensität. Die ETP (Kurve 3, Abb. 3) zeigten ein ähnliches Verhalten, doch mit noch geringerer Intensität.

Setzte man der BH-Mitochondrien-Suspension $0,3 \% \mathrm{Na}$-Desoxycholat zu, so entstand eine klare Lösung, deren Strahlungscharakteristik beim $\mathrm{O}_{2}$ Einleiten und Zusatz von Acridinorange der der RL-Mitochondrien ähnelte (Kurve 4, Abb. 3), nur daß ihre Intensität etwa $3 \%$ des Wertes der letzteren betrug.

Die Farbstoffe Eosin, Fluorescein und Methylenblau hatten keinen Einfluß. Die Wirkung von Acridinorange war unabhängig von Gegenwart oder Abwesenheit von Na-Succinat, 2.4-Dinitrophenol, Antimycin und Phenazinmethosulfat. KCN änderte auch an der kaum nachweisbaren natürlichen Strahlung nichts. EDTA *-Zusatz verminderte die Acridinorange-Strahlung deutlich, aber nicht wesentlich; dies könnte auf eine Beteiligung von SchwermetallIonen oder -Komplexen an der Lichterzeugung hinweisen.

Mit Aceton extrahierte RH-Mitochondrien-Suspensionen erzeugten innerhalb der Meßgenauigkeit keine Chemilumineszenz, ihre $\mathrm{O}_{2}$-Aufnahme war gleich Null. Bei Zugabe von Acridinorange trat das erste Maximum stark hervor (Abb. 4), der zweite langsam verlaufende Anstieg fehlte.

Der mit Aceton extrahierte Lipoidextrakt erzeugte als wäßrige milchig trübe Suspension beim Behandeln mit $\mathrm{O}_{2}$ keine Lumineszenz.

Da von Zhuralev und Mitarb. berichtet worden ist, daß Lipoidextrakte von humanen Geweben bei der Oxydation des Luftsauerstoffs in der Wärme Chemilumineszenz zeigen, wurden einige Modellversuche mit handelsüblichem Ei-Lecithin und SojaLecithin angestellt. Bei diesen ließ sich Chemilumineszenz feststellen, die - pro mg Substanz gerechnet - sehr viel stärker war als die der Mitochondrien.

* Äthylendiamintetraacetat. 


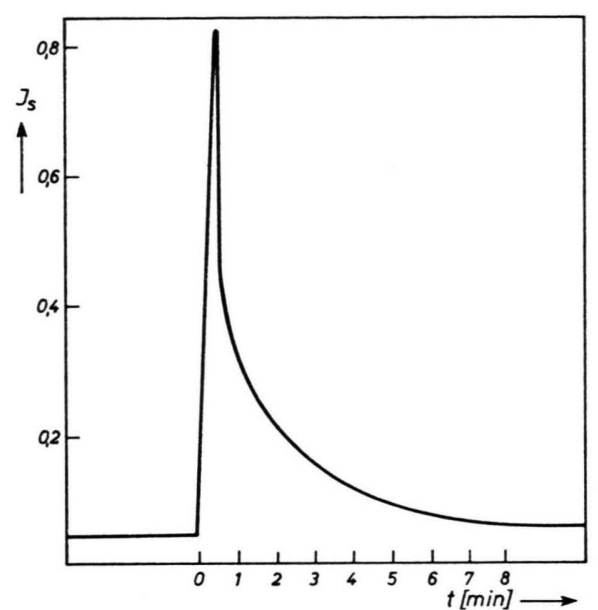

Abb. 4. Einfluß von Acridinorange $\left(1,8 \cdot 10^{-4}-m\right.$.) auf „phospholipoidfreie" $\mathrm{BH}$-Mitochondrien. Is = spezifische Intensität [Nanoampère/mg Totalprotein].

Die Chemilumineszenz trat bei Einleiten von $\mathrm{O}_{2}$ in Auflösungen des Lecithins auf und war unabhängig davon, ob das Lecithin in organischen Lösungsmitteln (Methanol oder Aceton) gelöst, in Wasser supendiert oder in Wasser durch NatriumDesoxycholat solubilisiert worden war. Acridinorange bewirkte im Gegensatz zu den MitochondrienSuspensionen hier keinerlei Verstärkung der Lichtintensität, wohl aber Eosin.

\section{Diskussion}

Die mitgeteilten Beobachtungen müssen zunächst zu dem Schluß führen, daß die Mitochondrien bei unseren Versuchsbedingungen im nativen Zustand mit großer Wahrscheinlichkeit keine Chemilumineszenz erzeugen. Erst bei einer Störung mechanischer bzw. physikalischer Art (Einfrieren-Auftauen, Ultraschall) oder chemischer Art (Zugabe von Acridinorange) wird bei $\mathrm{O}_{2}$-Behandlung eine mehr oder minder starke Lichterzeugung in Gang gesetzt. Es ergaben sich dabei deutliche Unterschiede zwischen den Mitochondrien der Rattenleber und des Rinderherzens; die Strahlung der letzteren war jeweils mindestens um eine Zehnerpotenz kleiner als die der ersteren, auch nahm die Strahlung bei BH-Mitochondrien durch Alterungseffekte bedeutend weniger zu als bei RL-Mitochondrien. Führt man diese Effekte auf morphologische und biochemische Ver-

18 F. S. Suöstrand, Nature [London] 202, 1075 [1964]. änderungen durch die erwähnten Einflüsse zurück, so sollten BH-Mitochondrien besonders widerstandsfähig sein.

Dies ergibt gewisse Parallelen zu den Ergebnissen von Sjöstrand und Mitarb. ${ }^{18}$, die durch elektronenmikroskopische Aufnahmen einen Einfluß des Suspensionsmediums auf die Struktur der verschiedenen Arten Mitochondrien beobachten konnten und dabei die gleichen Unterschiede fanden.

Die mechanische Wirkung von Ultraschall auf RLMitochondrien beruht nach Hogeboom und SchneI${ }_{\text {DER }}{ }^{19}$ auf der Fragmentierung der Membranen und Cristae und der Freisetzung von über $55 \%$ des Totalproteingehaltes in löslicher Form. Auch soll Ultraschall möglicherweise die G r e en schen „electron particles“ (EP) von den Oberflächen der Cristae der Mitochondrien „abpflücken“. Die partiellen Zerstörungen an den Mitochondrien beim Einfrieren und Auftauen ihrer Suspensionen sollen dagegen durch die Hypertonie der Rohrzuckerreste zwischen den Eiskristallen hervorgerufen werden.

Die Notwendigkeit, gewisse unzugängliche Bereiche der Mitochondrien aufzuschließen, ehe es zu einer Erzeugung von Licht kommt, kann auf verschiedenen Ursachen beruhen. Es kann beispielsweise das Substrat normalerweise nicht an den Ori gelangen, wo die Anregungsreaktion stattfindet, in diesem Fall wäre die Strahlung eine Indikation für einen unphysiologischen Vorgang, der erst nach partieller Zerstörung des Organs auftritt. Wenn es sich bei der angeregten strahlungserzeugenden Substanz um Sauerstoff (im Singulett-Zustand) handelt, würde es genügen, daß er auf dem Weg zum Reaktionsort (der Anregungsreaktion) bereits vorher, d. h. durch andere Reaktionen verbraucht worden wäre. Eine andere Möglichkeit bestünde darin, daß $\mathrm{O}_{2}$ wohl im normalen physiologischen Geschehen durch eine chemische Reaktion angeregt würde, seine Anregungsenergie jedoch, bevor es zu einer Lichtemission kommt, strahlungslos weitergibt (Löschreaktion). Wegen der relativ großen Lebensdauer der angeregten metastabilen Zustände des $\mathrm{O}_{2}$ ist ein solcher Mechanismus durchaus möglich, ja sogar wahrscheinlich. Die partielle Zerstörung der Mitochondrien würde jetzt die Löschreaktion des angeregten $\mathrm{O}_{2}$ stören oder verhindern, so daß dessen Anregungsenergie nun als Lichtemission freigesetzt wird.

19 „The Mitochondrion“, Editor A. L. Lehninger, A. W. Benjamin, Inc., New York, Amsterdam, S. 62. 
Der bei derartigen schwachen ChemilumineszenzErscheinungen entstehende im Singulett-Zustand angeregte $\mathrm{O}_{2}$ kann bei dem Übergang in den Grundzustand entweder selbst Licht emittieren - in der Regel in der Form von $\mathrm{O}_{2}-\mathrm{O}_{2}$-Excimeren - odei seine Anregungsenergie auf andere Moleküle übertragen. Falls diese fluoreszieren oder phosphoreszieren können - wie z. B. Acridinorange, geben diese die Energie in Form von Licht wieder ab.

Bei bestimmten Oxydations- und Reduktionsprozessen, an denen $\mathrm{H}_{2} \mathrm{O}_{2}$ beteiligt ist, kann SingulettSauerstoff im ${ }^{1} \Delta$ - oder ${ }^{1} \Sigma$-Zustand gebildet werden; bei der am besten untersuchten Reaktion zwischen $\mathrm{ClO}^{\ominus}$-Ionen und $\mathrm{H}_{2} \mathrm{O}_{2}$ entsteht ausschließlich $\mathrm{O}_{2}\left({ }^{1} 4\right)$, dessen Excimere eine Energie im Höchstfalle etwa $50 \mathrm{Kcal} / \mathrm{Mol}$ besitzen. Solche $\mathrm{O}_{2}$-Moleküle können den niedrigsten Triplett-Zustand des Eosins mit rund $49 \mathrm{Kcal} / \mathrm{Mol}$ anregen, was sich durch eine Verstärkung der bei der vorgenannten Reaktion auftretenden Chemilumineszenz zeigt. Acridinorange, dessen niedrigstes Energieniveau bei etwa $55 \mathrm{Kcal} /$ Mol liegt, wird von $\mathrm{O}_{2}\left({ }^{1} \Delta\right)$ nicht angeregt, wohl aber von $\mathrm{O}_{2}{ }^{\ominus}, \mathrm{O}_{2} \mathrm{H}$-Radikalen und von $\mathrm{O}_{2}\left({ }^{1} \Sigma\right)$.

So kann beispielsweise die Reaktion zwischen $\mathrm{Fe}(\mathrm{CN})_{6}{ }^{3 \ominus}$ und $\mathrm{H}_{2} \mathrm{O}_{2}$ Sauerstoff im ${ }^{1} \Sigma$-Zustand mit 34,5 Kcal/Mol erzeugen. Zwei $\mathrm{O}_{2}\left({ }^{1} \Sigma\right)$ liefern ausreichend Energie, um Acridinorange anzuregen ${ }^{* *}$.

Diese Erfahrungen geben einen Hinweis auf den möglichen Mechanismus der Mitochondrien-Lumineszenz. Macht man die Annahme, daß auch hier bei einer noch unbekannten Redox-Reaktion $\mathrm{O}_{2}$ im angeregten Singulett-Zustand entsteht, so ist es verständlich, daß intakte Mitochondrien praktisch keine Lumineszenz erzeugen, da der angeregte $\mathrm{O}_{2}$ sehr schnell durch sicher in großer Zahl vorhandene, den Anregungszustand löschende Substanzen strahlungslos in den Grundzustand überführt wird. Dabei

\footnotetext{
** Ob die Úbertragung der Reaktion durch Addition eines Sauerstoffs an den Farbstoff und nachfolgendem Zusammenstoß mit einem zweiten erfolgt oder ob sich zunächst ein $\mathrm{O}_{2} \mathrm{O}_{2}$-Excimeres bildet, das als solches seine Energie auf die Farbstoffe überträgt, wird z. Z. untersucht.
}

bleibt die Frage zunächst offen, ob, wie bereits oben formuliert, die Anregungsenergie in einem für die Funktion der Mitochondrien bedeutungsvollen Vorgang ausgenutzt wird.

Werden die Mitochondrien partiell zerstört, erhält der angeregte $\mathrm{O}_{2}$ Gelegenheit, seine Energie in Form von Licht abzugeben, wobei dessen Intensität mit zunehmender partieller Zerstörung der Struktur zunächst anwächst. Die Versuche mit „behandelten“ RL-Mitochondrien können allerdings nicht beweisen, $\mathrm{da}$ ß der als angeregte Substanz zu vermutende Singulett-Sauerstoff auch bei intakten Mitochondrien auftritt. Es ist nicht auszuschließen, daß bei der partiellen Zerstörung erst eine Reaktion in Gang kommt, die - vielleicht über die Bildung von $\mathrm{H}_{2} \mathrm{O}_{2}$ - auch den Singulett-Sauerstoff erzeugt.

Anders scheint es bei den BH-Mitochondrien zu sein, die in unbehandelter Präparation die langsam ansteigende und langandauernde Lumineszenz bei Zugabe von Acridinorange erzeugen. Da dieser Farbstoff gleichzeitig die $\mathrm{O}_{2}$-Aufnahme herabsetzt, könnte es sein, daß Singulett-Sauerstoff als Zwischenprodukt der Oxidation auftritt und dieser nach Abgabe seiner Energie an das Acridinorange die ihm zukommende Funktion nicht mehr ausüben kann.

Die hier beschriebenen Lumineszenz-Erscheinungen scheinen mit den von Vladimirov und Mitarb. gemachten Beobachtungen nicht übereinzustimmen. Es muß daher vermutet werden, daß dies an den in beiden Fällen verschiedenen Versuchsbedingungen liegt. Jedenfalls muß die Lipoid-Peroxidation als Erklärung für die von uns beobachteten Phänomene ausgeschlossen werden, da die im Zusammenhang damit auftretende Chemilumineszenz nicht durch Acridinorange sensibilisiert werden kann. Andererseits könnten beide Phänomene auf den gleichen Primärvorgang zurückzuführen sein, der sich unter verschiedenen Versuchsbedingungen jeweils in anderer Form äußert.

Der Deutschen Forschungsgemeinschaft und dem Fonds der Chemie danken wir für finanzielle Unterstützung. 